\title{
Mode Matching Interface for efficient coupling of light into Planar Photonic Crystals
}

\author{
Jeremy Witzens, Thomas Baehr-Jones, Michael Hochberg, and Axel Scherer \\ Department of Electrical Engineering, California Institute of Technology, Pasadena, California 91125 \\ witzeñs@caltech.edu
}

\begin{abstract}
We enhance the insertion efficiency from a silicon slab into a planar photonic crystal with a mode-matching interface consisting of a multi-layered diffraction grating ( $9 \%$ to $84 \%$ ). Computations are performed with 3D finite difference time domain.

@2003 Optical Society of America

OCIS codes: (230.3990) Microstructure devices; (050.1970) Diffractive optics
\end{abstract}

Anomalous dispersion properties of photonic crystals $[1,2]$ such as ultra-refraction and negative refraction [3-6] have been intensely studied in the past years and have resulied in applications such as the superprism effect [7-9] and self-collimation [10]. In particular, the superprism effect has been singled out as a good candidate to develop a nèw generation of planar lightwave circuits for frequency domain demultiplexion. One of the crucial difficulties in using the superprism effect for practical planar demultiplexers is the coupling of light into the photonic crystal with acceptable insertion losses. Although this is a crucial difficulty, it has received very limited attention in the previous literature [11]

Equi-frequency contours of photonic crystals have features such as approximately flat sides and cusps. The group velocity of Bloch modes is normal to the equi-frequency contour, so that in the vicinity of the cusp, the angular deflection in propagation direction corresponding to a small change in $\mathbf{k}$-vector is much higher than in an unpatterned slab.

At the cusps of equi-frequency contours, Bloch modes have a spatial Fourier structure dominated by two higher order Fourier components (figure 1) so that an incoming slab mode has very low insertion efficiency in the absence of mode matching. Multiple rows of holes, each row acting as a diffraction grating, compose a multi-layered grating (MLG) and generate the higher order Fourier components from the slab mode. The pitch of the gratings is $\sqrt{2} a$, where $a$ is the lattice constant of the planar photonic crystal (PPC). The distance $d$ between rows is chosen so that contributions of successive gratings to the higher diffraction orders interfere constructively and so that reflections from successive gratings interfere destructively.

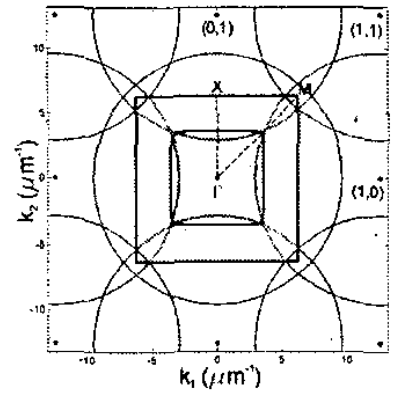

(a)

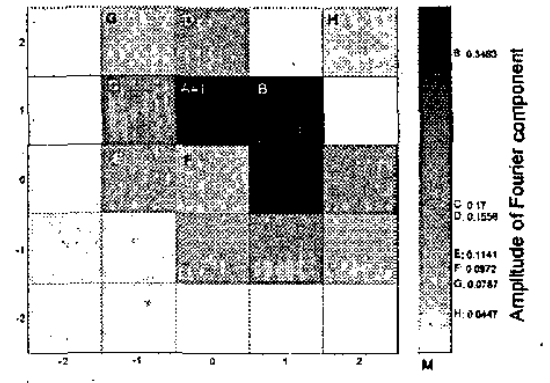

(b)

Fig. 8. (a) Equi-frequency contour of the $2^{\text {nd }}$ Band of a square lattice PPC (squarish black contour). The PPC is defined in a $205 \mathrm{~nm}$ thick Silicon slab clad with $\mathrm{SiO}_{2}$ and has a lattice parameter of $0.5 \mu \mathrm{m}$ and a hole radius of $0.15 \mu \mathrm{m}$. The black square represents the $I^{\text {st }}$ Brillouin zone $(\mathrm{BZ})$. The circle centered on $\Gamma$ represents the equi-frequency contour of the unpatterned slab and the other circles represent the higher order Fourier components generated by the array of holes. (b) Fourier decomposition of a Bloch mode of the $2^{\text {nd }}$ Band. The Bloch mode is on $\Gamma M$ and corresponds to the free space wavelength $1.55 \mu \mathrm{m}$. The axes give the offset of the component from the $1^{\text {s }} \mathrm{BZ}$ in integer multiples of the inverse lattice vectors. The two dominant Fourier harmonics (A) correspond to harmonics $(1,0)$ and $(1,0)$ in $(a)$.

We compute the insertion efficiency with finite difference time domain (FDTD) simulations (figure 2). Field probes store the field at regular intervals along the propagation direction. After applying a Fourier transform to the 
time-series, the following functional is used as an inner product between stored field profiles $(e, h)$ and the mode profiles $(\hat{e}, \bar{h})$ of various diffraction orders [12]:

$$
\int_{A}(e \times \bar{h}+\vec{e} \times h) \cdot \hat{z} \cdot d A
$$

where $\mathrm{z}$ is the direction of propagation and $\mathrm{A}$ is a plane normal to $\hat{z}$. This way the insertion efficiency into the $2^{\text {nd }}$ band of a square lattice photonic crystal of lattice constant $a=0.47 \mu \mathrm{m}$ is shown to be enhanced from $9 \%$ to $84 \%$ with an MLG composed of 19 rows of $40 \mathrm{~nm}$ holes and a separation $d=1.24 \mu \mathrm{m}$ between rows. We also show that the $0^{\text {th }}$ diffraction order is essentially reflected at the interface from the MLG to the PPC and that the $1^{\text {st }}$ order is essentially transmitted (figure 3 ).

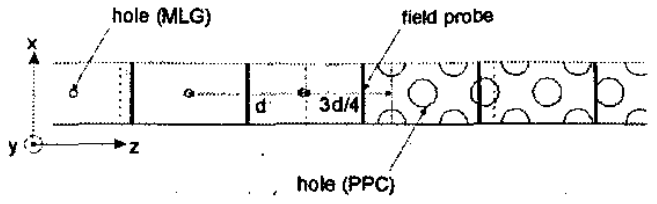

Fig. 2. Setup of the computational domain. The interface is along $\mathrm{x}, \mathrm{y}$ is the out-of-plane direction and $\mathrm{z}$ is the direction of propagation. Bloch boundary conditions are imposed along $x$ so as to effectively simulate an infinite PPC in the $x-$ direction. Probes are regularly spaced along the direction of propagation.
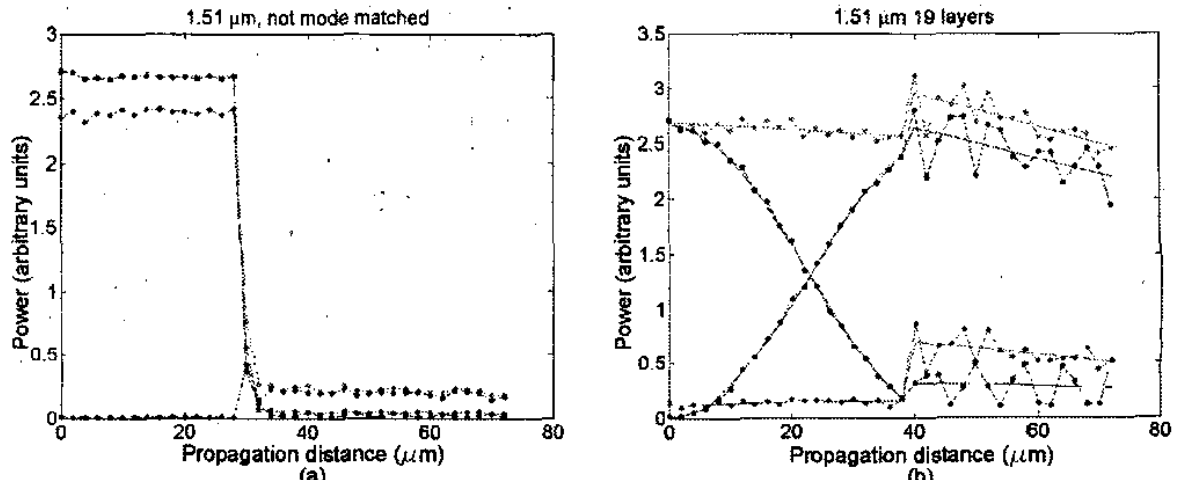

Fig. 3. Simulation results. The black curve shows the power contained in the original slab mode ( $0^{\text {th }}$ diffraction order), the blue curve shows the power contained in the higher diffraction orders 1 and -1 . The green curve shows the power contained in forward propagating slab modes and the red curve shows the power contained in backwards propagating slab modes. (a) Interface between the slab (left) and the non mode-matched PPC (right). Almost all the power is reflected at the interface. (b) Interface between the MLG (left) and the PPC (right). In the MLG the power is transferred from the $0^{\text {th }}$ diffraction order into the higher diffraction orders. There is a $84 \%$ transmission at the interface.

In conclusion we have shown that by generating the higher order Fourier components of the Bloch mode, a multilayered grating composed of several rows of holes can be designed to operate mode matching between an unpatterned slab and a planar photonic crystal. We have used an inner product to extract the intensities of all diffraction orders from field profiles obtained by 3D FDTD inside the MLG and the PPC.

[1] E. Yablonovitch, "Inhibited spontaneous emission in solid-state physics and electronics", Phys. Rev. Lett., 58, 2059-2062 (1987)

[2] T. F. Krauss, R. M. De La Rue, and S. Brand, "Two-dimensional photonic-bandgap structures operating at near infrared wavelengths", Nature, 383, 699-702 (1996)

[3] S.-Y. Lin, V. M. Hietala, L. Wang, and E. D. Jones, "Highly dispersive photonic band-gap prism", Opt. Lett., 21, 1771-1773 (1996).

[4] B. Gralak, S. Enoch, and G. Tayeb, "Anomalous refractive properties of photonic crystals", J. Opt. Soc. Am. A, 17, 1012-1020 (2000).

[5] H. Kosaka, T. Kawashima, A. Tomita, M. Notomi, T. Tamamura, T. Sato and S. Kawakami, "Superprism phenomena in photonic crystals", Phys. Rev. B - Rapid Comm., 58, R10096-R10099 (1998).

[6] H. Kosaka, T. Kawashima, A. Tomita, M. Notomi, T. Tamamura, T. Sato and S. Kawakami, "Self-collimating phenomena in photonic crystals", Appl. Phys. Lett., 74, 1212-1214 (1999).

[7] T. Baba, and D. Ohsaki, "Interfaces of Photonic Crystals for High Efficiency Light Transmission", Jpn. J. Appl. Phys., 40, 5920 -5924 (2001).

[8] A. W. Snyder, J. D. Love, Optical Waveguide Theory (Chapman \& Hall, 1983) Chap. 11. 